\title{
Nanoscale Conductivity Contrast by Scattering-Type Near-Field Optical Microscopy in the Visible, Infrared and THz Domains
}

\author{
Fritz Keilmann • Andreas J. Huber • Rainer Hillenbrand
}

Received: 15 February 2009 / Accepted: 19 May 2009 /

Published online: 5 June 2009

(C) The Author(s) 2009. This article is published with open access at Springerlink.com

\begin{abstract}
We demonstrate the wide application potential of optical near-field microscopy for mapping samples with locally varying conductivity. The apertureless, scattering-type optical near-field microscope (s-SNOM) operates on an AFM basis with an added lightscattering channel, wherein a standard cantilevered tip suffices to accomodate the full spectral range from visible to $\mathrm{THz}$ frequencies. The optical maps appear in amplitude and phase contrast, simultaneously with the topography map, at a spatial resolution of typically $20 \mathrm{~nm}$. Visible and near-infrared operation is best suited for distinguishing metals, while a sensitive response to semiconductors is ideally provided with infrared and $\mathrm{THz}$ operation. Various types of conductivity spectral features can be explored in specific regions, corresponding to the elementary excitation linked to e.g. superconductivity, electron correlation, or low-dimensional conduction.
\end{abstract}

Keywords Metallic conduction · Semiconductors · Correlated conductors ·

Conductivity contrast · Near-field optical microscopy · Visible near-field microscopy ·

Infrared near-field microscopy $\cdot \mathrm{THz}$ near-field microscopy

\section{Introduction}

Spectroscopy in the infrared and $\mathrm{THz}$ regions has been an important and successful extension of the traditional visible-light spectroscopy for the characterization of condensed matter. The reason is that light-matter interaction in these long-wavelength regions can sensitively modify light transmission, reflection or scattering with characteristic spectral

F. Keilmann $(\bowtie)$

Max Planck Institute for Quantum Optics and Center for NanoScience, 85714 Garching, Germany e-mail: fritz.keilmann@mpq.mpg.de

A. J. Huber

Max-Planck-Institut für Biochemie, 82152 Martinsried, Germany

A. J. Huber $\cdot$ R. Hillenbrand

Nanooptics Laboratory CIC nanoGUNE Consolider and Center for NanoScience,

20018 Donostia-San Sebastián, Spain 
features that are often resonances. Best-known examples are the absorption lines due to molecular vibrations which are characteristic for any chemical compound, whence the midinfrared range from 3 to $30 \mu \mathrm{m}$ is often referred to as the "chemical fingerprint" region. Less well known is the fact that conducting electrons and quasiparticles also induce characteristic spectral features such as plasma edges which lie in the infrared and $\mathrm{THz}$ regions, respectively, for highly and lowly conducting semiconductors; such spectra can be used to extract conductivity-related quantities such as carrier density and carrier mobility; for example, in the near-infrared and visible regions, interband excitations of mobile carriers dominate the spectra of highly conducting metals.

Near-field optical techniques have enabled the optical mapping of sample surfaces at spatial resolutions no longer limited by the wavelength $\lambda$ [1]. This innovation has enabled a particularly big step forward in the long-wavelength infrared and $\mathrm{THz}$ spectral regions where far-field microscopy could never have provided any sub-micrometer spatial resolution. Indeed sub-micrometer resolution has become possible even for microwaves, by the principle of scattering the probing light from a sharp metal tip, the so-called apertureless or scattering-type optical near-field microscopy, or s-SNOM [2-4]. This paper reviews the s-SNOM's achievements to characterize local conductivity, presents a description of some basic mechanisms, and suggest its future extension in respect to frequency coverage and scientific application.

\section{Experimental technique}

The heart of s-SNOM is the tip used for light scattering. Metallic tips have been mostly employed because they can exhibit a high field confinement at the apex, and because the metal shaft enables antenna effects that can improve the coupling between the near field and a tightly focused laser beam [5]. The most popular scanning probe platform has been the AFM (Fig. 1) because it readily allows to probe non-conducting sample material, and furthermore, because its cantilever very naturally allows to modulate the tip-sample separation $z$ at the cantilever resonance frequency $\Omega$ which, conveniently, lies in the range between $10 \mathrm{KHz}$ and $1 \mathrm{MHz}$. This modulation has been established to be essential for obtaining a pure near-field response that is not affected by light scattering from remote parts of the shaft and cantilever structure that are also unavoidably exposed to the illuminating beam. When to this effect $z$ is sinusoidally varied between 0 and about 20 or $40 \mathrm{~nm}$, a distance given by the typical range of the near-field which is roughly equal to the tip's radius of curvature $a$, this motion strongly modulates the near-field part of the scattering signal. Consequently, this part can then be preferentially filtered from the "background" scattering by processing the detector signal through a synchronuous amplifier locked either to the oscillation frequency $\Omega$ or even better, its second or third harmonic [4].

\section{Theoretical response of conductive samples in the near-field microscope}

Mobile charge carriers can follow electric fields up to high frequency limits given by the nature of the host material. For example, electric currents in metals can be excited up to $>1000 \mathrm{THz}=1 \mathrm{PHz}$ in the ultraviolet, whereas a frequency limit of $1 \mathrm{THz}$ may apply to a lowly doped semiconductor. Near such a limit the spectral response-expressed in the complex dielectric function of the material, $\varepsilon=\varepsilon_{1}+\mathrm{i} \varepsilon_{2}$ - exhibits prominent features which are widely applied for characterizing the conductance mechanism [7]. The near-field 
response, on the other hand, has been shown to depend on the local dielectric function of the sample material. For a given geometry and material of the tip, and for numerous types of sample materials, there is a one-to-one relationship between a sample's local value $\varepsilon_{1}+$ $\mathrm{i} \varepsilon_{2}$ and the complex scattering signal consisting of amplitude and phase, $s \mathrm{e}^{\mathrm{i} \phi}$. The pointdipole/image-dipole model of near-field interaction gives a simple expression to approximately describe this relationship [4].

We use this model here to demonstrate the essential spectral features to be expected in the spectroscopic near-field microscopy of conducting materials. For this examplaric calculation we consider three simple, hypothetical, Drude-model materials which are characterized by only three quantities, the high-frequency dielectric constant $\varepsilon_{\infty}$, the plasma frequency $\Omega_{p}$, and the mean time $\tau$ between carrier collisions

M, a metal: $\tau=10^{-15} \mathrm{~s}, \Omega_{p}=10^{16} \mathrm{~s}^{-1}$;

$\mathrm{H}$, a highly doped semiconductor: $\tau=310^{-13} \mathrm{~s}, \Omega_{p}=10^{14} \mathrm{~s}^{-1}$;

L, a lowly doped semiconductor: $\tau=310^{-12} \mathrm{~s}, \Omega_{p}=310^{12} \mathrm{~s}^{-1}$.

Materials $\mathrm{H}$ and $\mathrm{L}$ idealize semiconductors with mobile carrier concentration in the order of $n=10^{19} \mathrm{~cm}^{-3}$ and $10^{16} \mathrm{~cm}^{-3}$, respectively. We obtain the dielectric functions as sums of $\varepsilon_{\infty}($ set $=1$ for $M$, and $=16$ for $H, L)$ and the Drude term,

$$
\begin{aligned}
& \varepsilon_{1}(\omega)=\varepsilon_{0}-\Omega_{p}^{2} /\left(\omega^{2}+1 / \tau^{2}\right), \\
& \varepsilon_{2}(\omega)=\Omega_{p}^{2} /\left(\omega \tau\left(\omega^{2}+1 / \tau^{2}\right)\right) .
\end{aligned}
$$

These functions (see Fig. 2a) are inserted into

$$
s e^{i \varphi} \propto \alpha /\left(1-\alpha \beta /\left(16 \pi(a+z)^{3}\right)\right)
$$

which is a simplified expression from the dipole model of near-field interaction $[8,9]$ (cf. discussion of eq. 15 of reference [10]), where $\alpha$ is the point-dipole polarizability

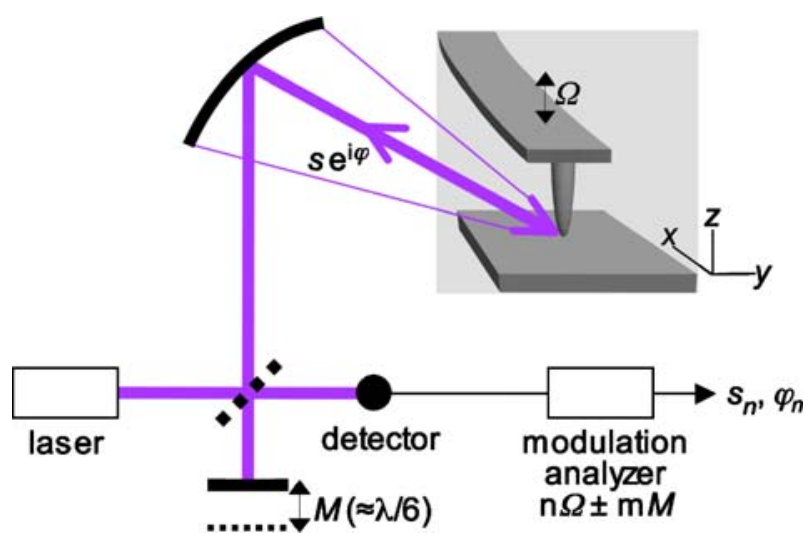

Fig. 1 Sketch of s-SNOM. The basic AFM is operated with its tip-sample separation $z$ modulated at frequency $\Omega$. Backscattering of an applied laser beam is detected interferometrically, in the sketched example using a Michelson configuration and a phase modulation at frequency $M$ for electronic sideband filtering (pseudoheterodyne detection) [6]. 
representing the tip, and $\beta=(\varepsilon-1) /(\varepsilon+1)$ is the surface response function representing the sample. The results are the amplitude and phase spectra of near-field contrast of L, H and M (Fig. 2b, c), which we compute here relative to a (hypothetical, similar to diamond) highindex dielectric material characterized by a constant $\varepsilon_{r}=6$ for all frequencies. For simplicity, we use a constant value $\alpha$ corresponding to a (hypothetical, similar to $\mathrm{Pt}$ in the mid infrared) tip material having $\varepsilon_{r}=-1310+760 \mathrm{i}$, throughout this paper. Furthermore, we assume a tip oscillation amplitude $\Delta z \approx a$, and a demodulation at $2 \Omega$.

The near-field contrast thus exhibits spectral changes where the conductivity approaches its high-frequency limit, i.e., near $\Omega_{p}$ where $\varepsilon_{1}$ changes sign. This is as in polar crystals which exhibit a marked near-field resonance also where $\varepsilon_{1}$ changes sign, due to a phononic
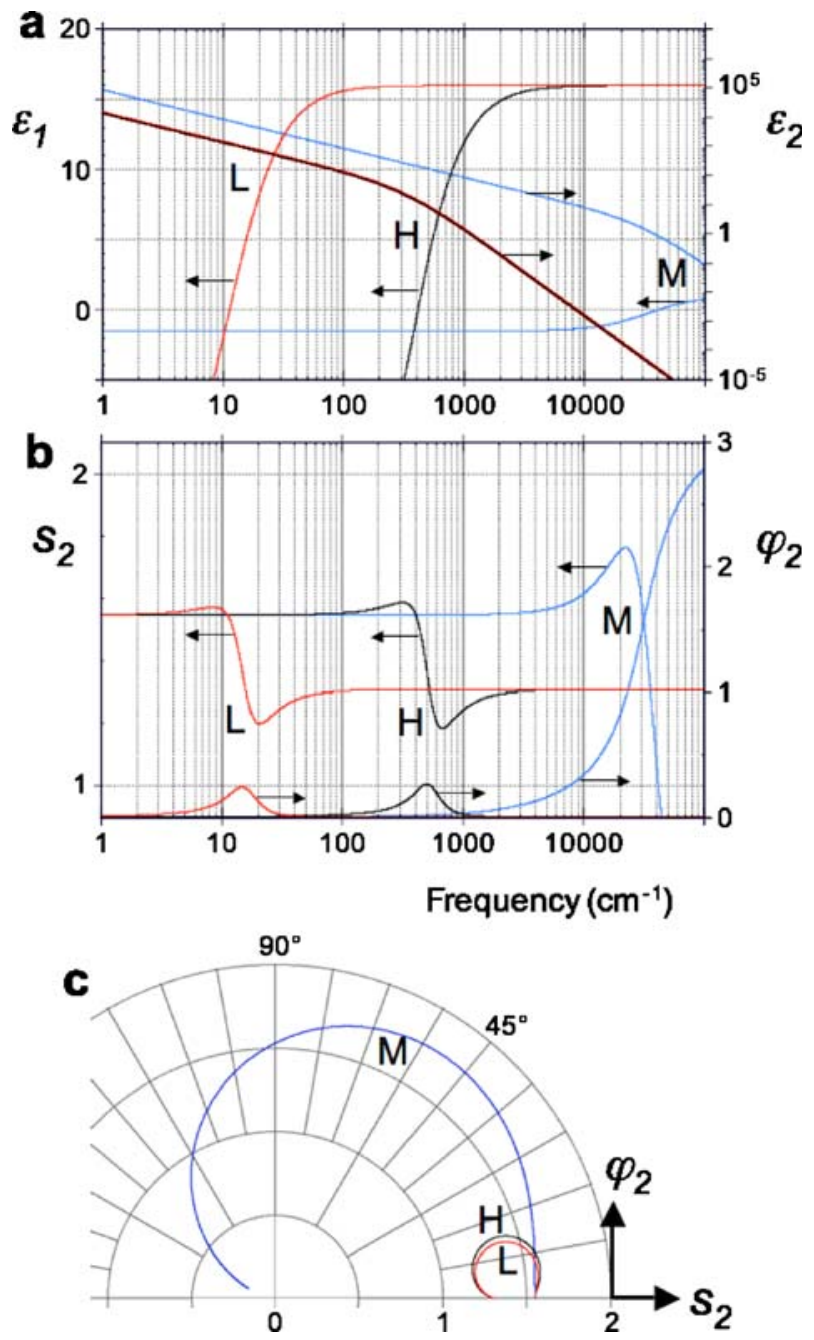

Fig. 2 Dielectric functions (a), and predicted near-field contrast spectra (b, c) relative to a dielectric sample with $\varepsilon_{r}=6$, for three hypothetical Drude conductors: a metal ( $\mathrm{M}$, blue), a highly (H, black) and a lowly (L, red) doped semiconductor. The near-field spectra $(b, c)$ are calculated from the point-dipole model, in amplitude and phase; when displayed in polar coordinates the spectra describe curved trajectories which are, as a function of rising frequency, traversed anti-clockwise. 
"Reststrahlen" band of negative $\varepsilon_{1}$ [11]. Here, to concentrate on the effects of charge carriers, our model materials are chosen to have no optically active phonons.

Far below $\Omega_{p}$ the near-field amplitude of all three conductors comes out constant, $55 \%$ above that of the $\varepsilon_{r}=6$ reference. This means that s-SNOM at low frequency can recognize the presence of a conductor but it cannot distinguish between different pure Drude conductors, even not when they differ in $\varepsilon_{\infty}$. Also, the phase of scattering at frequencies far below $\Omega_{p}$ is the same for different Drude conductors and equal to that of any reference dielectric. At probing frequencies far above $\Omega_{p}$ there is no information on conductivity: the scattering amplitude becomes a constant which lies above or below the reference value depending only on the value of $\varepsilon_{\infty}$ relative to $\varepsilon_{r}$.

Clearly for Drude conductors, the near-field probing of conductance requires to operate the s-SNOM within an order of magnitude around $\Omega_{p}$. The spectral near-field response feature resembles that of a relaxator with a slight indication of resonance enhancement. Stronger enhancement could, of course, be expected if a smaller damping would apply, as evident from the phonon case [11]. The sensitivity range of one order of magnitude in frequency corresponds, due to $\Omega_{p} \propto n^{2}$, to a sensitivity bandwidth of two orders of magnitude in mobile electron concentration. Thus for locally quantifying a Drude conductor with a doping variation up to two orders of magnitude it could just suffice to operate the sSNOM at a single frequency. A multi-decade wide variation of $n$, however, would necessitate operating at several frequencies spaced maximally $10 \mathrm{x}$ apart.

\section{Apertureless near-field microscopy development from microwave to visible}

The first SNOM used an aperture and was realized in the microwave region [12], while one early SNOM with an apertureless tip operated even at radio frequencies [13]. The apertureless microwave SNOM [14] allowed to demonstrate a microwave near-field contrast from a structured metal film. As common with microwave instrumentation both amplitude and phase were recorded, and thus yielded complex-valued information about the light-matter interaction. Figure 3 shows an example in which a sub-micrometric resolution is achieved.

Present microwave SNOMs feature an enhanced sensitivity because they employ a microwave resonator close to the tip. This yields a frequency shift and a quality factor as the two observables. Common to all microwave SNOMs is that no free-space beam is involved in the coupling to the tip, rather, coaxial or coplanar transmission lines are used for this purpose [15]. Microwave SNOMs have the demonstrated capability of quantifying the local conductivity of lowly doped semiconductors over at least two orders of magnitude, at a spatial resolution of $100 \mathrm{~nm}$ [16], and better [17].

The first apertureless s-SNOM operated at $633 \mathrm{~nm}$ wavelength to image transparent dielectric materials [18]. Metallic materials were investigated with the first heterodyne interferometric s-SNOM [9] that resulted, for the scattering contrast of $\mathrm{Au}$ relative to $\mathrm{Si}$, in an amplitude ratio of $2.5 \pm 0.1$, and a phase difference of $60^{\circ} \pm 10^{\circ}$. Nanostructured metal samples have since served as a standard in s-SNOM testing [19-24].

A systematic s-SNOM study of different metals has hitherto not been done although it is in the near infrared, in the visible and beyond where optical contrast between different metals should be highest, and where therefore s-SNOM could best be applied to analyze nanocomposites formed out of different metals. To emphasize this potential we predict nearfield spectra of three metals (Fig. 4), using the dipole model Eq. 3 and published dielectric functions. The latter are - for all metals - grossly different from a simple Drude result, 

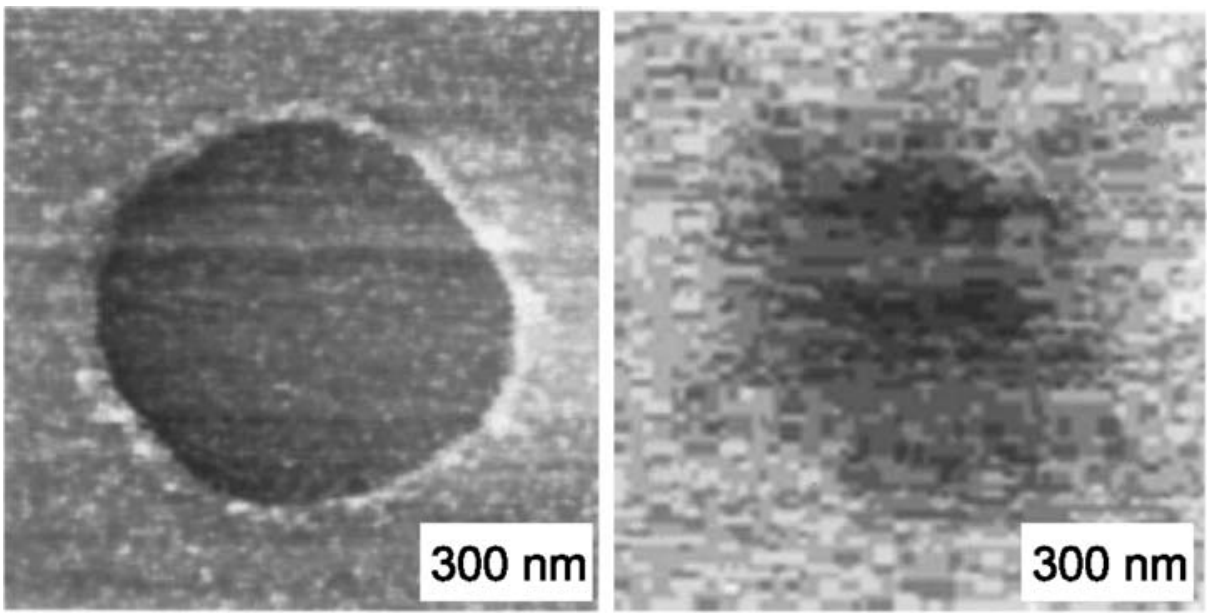

Fig. $31 \times 1 \mu \mathrm{m}^{2}$ near-field image obtained with a microwave SNOM [14] operated at $1.38 \mathrm{GHz}$; the sample is a $2 \mathrm{~nm}$ thick PT/C film structured by a $700 \mathrm{~nm}$ wide hole as seen in the topography image (left); the microwave transmission amplitude image (right) reveals that the microwave near-field resolution achieved is about $200 \mathrm{~nm}$.

owing to the contribution of interband excitations of mobile electrons, and also to the possible frequency dependence of the quasiparticle mass and of $\tau$.

Clearly the non-Drude contributions dominate in the near-field spectra of metals and induce very prominent features where $\varepsilon_{1}$ crosses zero. The overall near-field spectra become much richer in these non-Drude cases. This will certainly open new possibilities for studying the physics of metal transport. For such studies the realization of a broadband sSNOM operating in the near-infrared, visible and ultraviolet spectrum becomes highly desirable, and could be achieved by implementing coherent continuum sources or coherent frequency-comb beams, as recently shown in the mid infrared [26]. Note that broadband sSNOM determination of the complex dielectric function could valuably complement spectroscopic-ellipsometry measurements which are limited in spatial resolution by freespace diffraction. On the other hand, it is mandatory to use the most reliable dielectric data when predicting near-field spectra. A critical evaluation of the presently available data of metal optical constants is, however, beyond the scope of our present contribution, and therefore, the examples given in Fig. 4 can only be regarded as an approximation which, however, fully serves for illustrating the basic usefulness of a future s-SNOM research on metals in the near-infrared, visible and ultraviolet regions.

\section{Mid-infrared near-field microscopy of highly-doped semiconductors}

Much of the advancement of s-SNOM has been attained using the $\mathrm{CO}_{2}$ and $\mathrm{CO}$ lasers, in the $9-11 \mu \mathrm{m}$ and 5-6 $\mu \mathrm{m}$ wavelength regions, respectively $[5,6,8,10,11,20,21,24,27-$ 37]. This came first of all because mid-infrared can be used to exploit chemical fingerprints for recognizing chemical composition, now at nanometric resolution $[8,31,33,37,38]$, but furthermore for technical reasons. Compared to the visible and near infrared regionswhere also simple lasers with ideally 1 to $10 \mathrm{~mW}$ c.w. beam power are available - the mid infrared has turned out to be superior in allowing the experimenter to observe and stabilize 

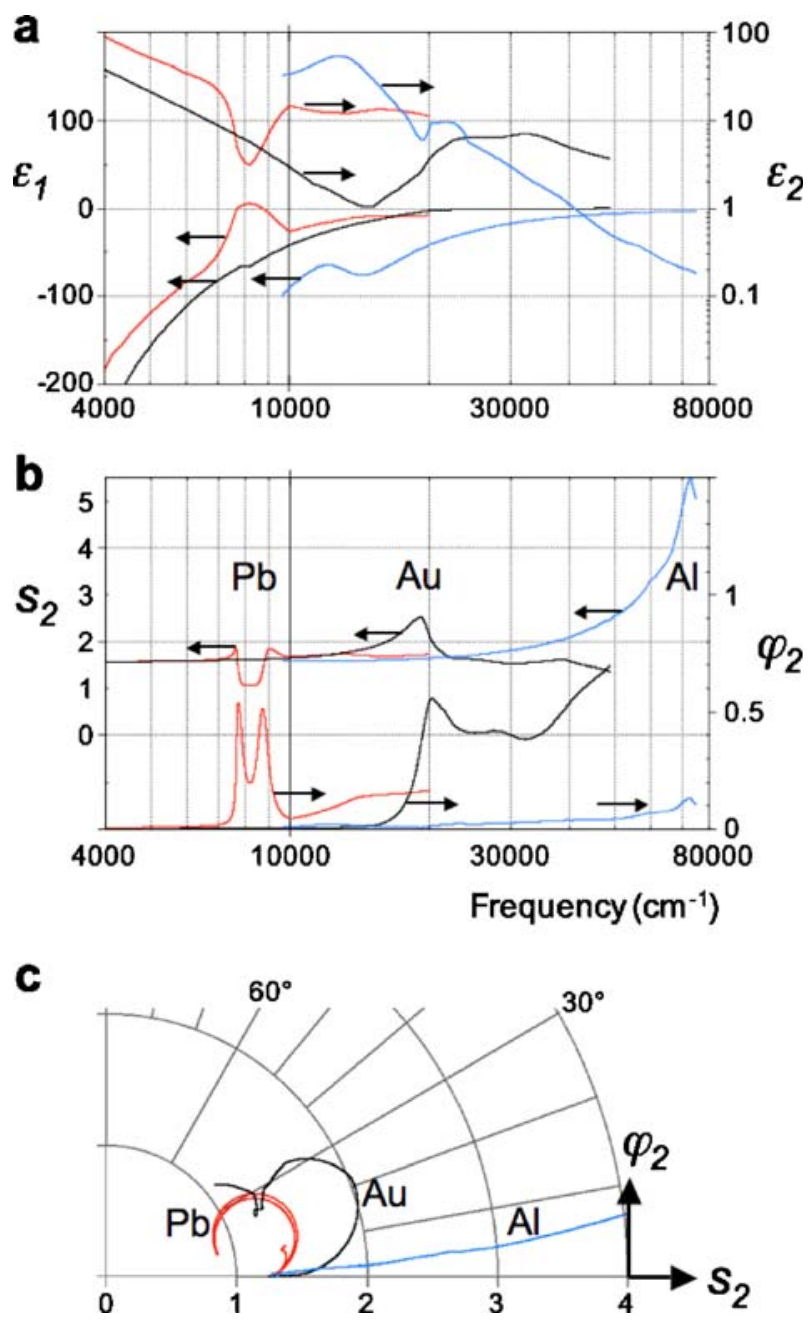

Fig. 4 Dielectric functions (a) of three real metals $\mathrm{Pb}, \mathrm{Au}$ and $\mathrm{Al}$, obtained from ref. [25], and predicted near-field contrast spectra $(b, c)$ relative to a dielectric reference with $\varepsilon_{r}=6$, as calculated from the pointdipole model, in amplitude and phase, and displayed also in polar coordinates.

the near-field interaction, and to take reproducible optical images. One reason for this lies in the longer wavelength which makes the interferometric phase measurement less vulnerable to mechanical vibrations. Another distinct advantage may be a better coupling between the tip-confined near-field and an external focused beam, possibly due to improved antenna efficiency because the mid-infrared wavelength approximately matches the typical length $(20 \mu \mathrm{m})$ of commercial AFM tips [5].

An example of mid-infrared s-SNOM imaging displaying a pure conductivity effect is shown in Fig. 5. The sample was prepared to have $250 \mathrm{~nm}$ wide stripes of high doping, at a $400 \mathrm{~nm}$ period. This doping induced a minute topography depression of $0.1 \mathrm{~nm}$ [28], which could not by itself lead to a noticeable optical effect. The images display the regions of conductivity in distinct infrared contrast. Especially noteworthy is the fact that this contrast 
reverses sign when the wavelength is changed from 9.3 to $10.6 \mu \mathrm{m}$. This observation is as predicted from the theoretical behaviour in the vicinity of the the plasma frequency $\Omega_{p}$, and in fact this observation helped verify the dipole model. Consider again Fig. 2b which shows the typical plasmon resonance of a highly doped semiconductor $(\mathrm{H})$, here at about $500 \mathrm{~cm}^{-1}$. Within the sharp decrease of the scattering amplitude a most characteristic point is either where the amplitude reaches (i) the level of the pure host material, or (ii) the level of the reference material. Depending on which of these materials one chooses to reference the near-field contrast to, point (i) or (ii) marks the spectral position $\lambda_{c}$ where the image contrast will flip its sign. In the case of Fig. 5 the reference is undoped Si whence (i) applies, and $\lambda_{c}$ lies between the two wavelengths used.

Further work with mid-infrared s-SNOM has confirmed that the local conductivity contrast can indeed be quantified along a doping gradient, by imaging a series of real-world nanostructured device samples produced by microelectronics industry [34]. For this a sample containing transistor elements was prepared by polishing, and SEM was used to visualize the position of the different elements of the nanocircuit. When viewed in the midinfrared s-SNOM, the topography image hardly showed any of these elements, but rather exhibited considerable topography variation from imperfect polishing. Yet in the infrared

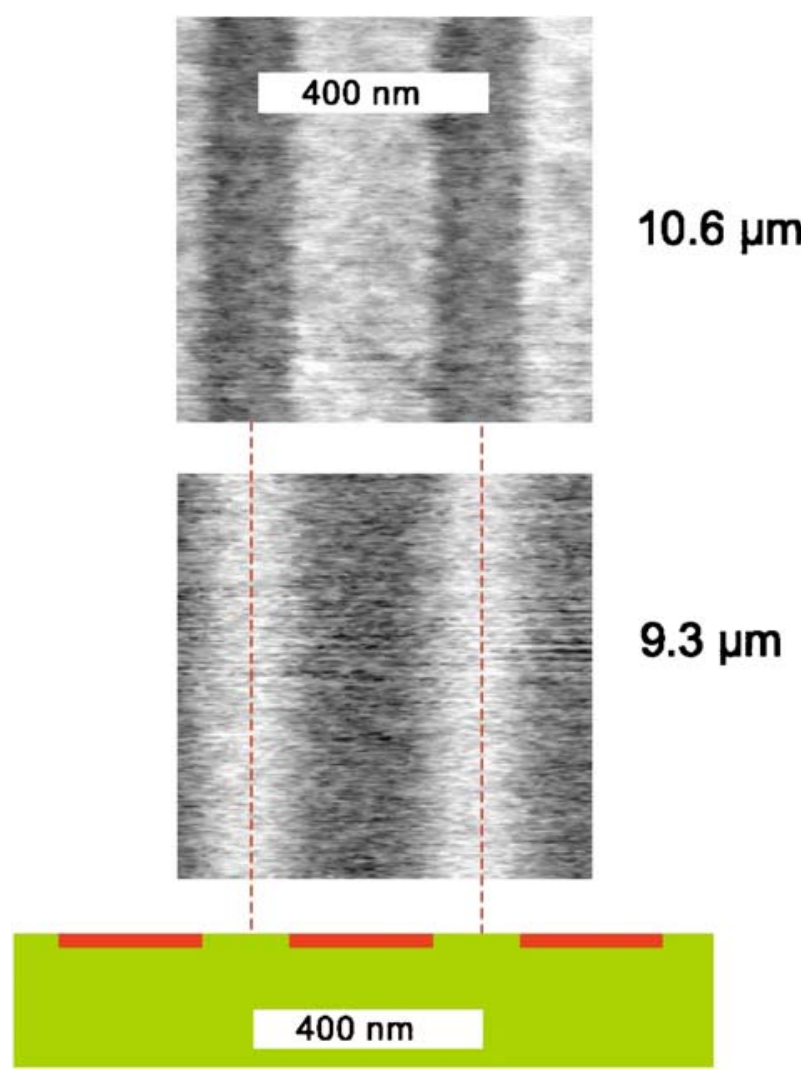

Fig. $5800 \times 800 \mathrm{~nm}^{2}$ mid-infrared s-SNOM images of a flat Si sample with periodic stripes of high doping (nominal doping level $n=510^{19} \mathrm{~cm}^{-3}$, extending $30 \mathrm{~nm}$ into the crystal, as indicated in red in the bottom sketch); notice the contrast reverses at a wavelength between the two indicated and used for these images. 
image the contact elements and other material features were seen very clearly, with phase and amplitude contrasts characteristic of these materials (Al, Ti, TiN, $\mathrm{Si}, \mathrm{Si}_{3} \mathrm{~N}_{4}$, and $\mathrm{SiO}_{2}$ ), at negligible crosstalk fom the topography. An example of such an image will be shown and discussed in the following section (Fig. 7a).

Here we show in Fig. 6 the near-field amplitude spectrum taken from a specific location of a highly doped Si sample. The spectrum was obtained by repeatedly taking an s-SNOM image at different laser frequencies, and then extracting the near-field contrast in reference to a thin Au patch deposited on the sample. The resulting data points proove a rise of the amplitude signal with decreasing frequency. They fit well with a theoretical curve calculated from the finite dipole model [10]. Furthermore, the curve in Fig. 6 has taken the concentration dependence of $\tau$ (i.e., of the mobility) into account [39], with the resulting fit parameter $n=410^{19} \mathrm{~cm}^{-3}$ that agrees well with the design electron concentration [40].

The scatter of the experimental data in Fig. 6 is probably owed to an obvious moment of non-repeatability in quantitative s-SNOM: when repeatedly recording an image one can often notice a slight drift of the sample; also an abrasion or other deterioration of the probing tip might occur; and when changing the wavelength minute beam directional changes might happen [33]. To overcome these effects spectroscopic s-SNOM has been initiated in which a broad band of frequencies is applied and read out simultaneously, such that a near-field spectrum is obtained at each pixel while scanning [26, 41].

As already mentioned, a doping gradient can be determined from just a single near-field image [34]. The extraction of the scattering contrast along the gradient results in resonance curves akin to those in Fig. $2 \mathrm{~b}(\mathrm{H})$. In polar coordinates, the complex scattering response describes more than half a circle (Fig. 3b in [34]). The fair agreement between model and observation is another proof that the s-SNOM's contrast mechanism has been basically understood.

A very interesting application of s-SNOM carrier density mapping has already been recently demonstrated: local conductivity in strained semiconductors including the technologically important $\mathrm{Si}$ [40]. In this study the complex near-field contrast was mapped in the neighborhood of permanent indents impressed by a sharp diamond tip, over the $890-1090 \mathrm{~cm}^{-1}$ frequency range. The results are very impressive, especially, because even the carrier mobility could be extracted ontop of the carrier concentration.

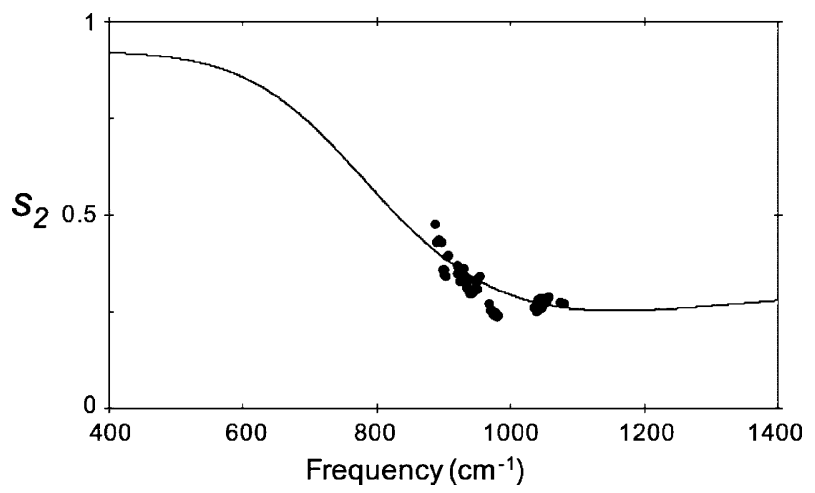

Fig. 6 Near-field spectrum of $s_{2}$ amplitude contrast between a given location on a highly doped Si sample, and an $\mathrm{Au}$ patch deposited nearby. The data points were extracted from a number of s-SNOM images taken consecutively at different wavelengths. The curve is from theory [40]. 


\section{$6 \mathrm{THz}$ near-field microscopy to quantify low doping in semiconductors}

As is clear from Fig. 2 any imaging of conductivity contrast in lower doped semiconductors requires that the s-SNOM is operated with far-infrared radiation. Accordingly, development of s-SNOM in the few $\mathrm{THz}$ region was initiated by employing the "time-domain" $\mathrm{THz}$ spectrometer where a train of sub-ps near-infrared pulses is used for both the generation of broadband $0.1-7 \mathrm{THz}$ pulses, as also for their time-resolved sampling via a mechanical delay stage. One study of metal stripes was interpreted to demonstrate $150 \mathrm{~nm}$ spatial resolution, nonwithstanding the fact that the image was taken at a $1200 \mathrm{~nm}$ high topographical edge and also appeared in a faint contrast of only $0.5 \%$ [42]. When a sample modulation was employed higher contrast was reported but at deteriorated resolution of $1800 \mathrm{~nm}$ [43].

In the first THz s-SNOM which employed the distance modulation technique described in sect. 2 clear evidence was established of attaining the pure near-field interaction: the contrast between materials was of the order of $100 \%$; the tip-sample approach induced a signal change compatible with the 200-300 nm size of the home-made tip used; and the spectra of near-field contrast agreed with the model prediction and distinctly distinguished a metallic from a lowly-doped semiconductor sample [41]. Note these near-field spectra were truely broadband, covering four octaves from 0.1 to $3 \mathrm{THz}$, but unfortunately the weak scattered signal (note the source emits typically several $\mu \mathrm{W}$ of $\mathrm{THz}$ power) necessitated 19 min observation time for each spectrum such that scanning an image was not practical.

A substantially different and highly successful approach has used a $10 \mathrm{~mW}$ gas laser beam at fixed $2.54 \mathrm{THz}$ frequency, and a standard metallized AFM tip with $30 \mu \mathrm{m}$ length of the tip shaft [44]. Although the wavelength $(118 \mu \mathrm{m})$ exceeds this length fivefold the nearfield signal was strong enough to be measured in $0.1 \mathrm{~s}$. This allowed high-quality scanned images which were taken, at about $10 \mathrm{~s} /$ line, of industrial nanostructured semiconductor device samples. Pure near-field interaction could be ascertained again by taking approach curves, and amplitude contrasts could be quantified. In Fig. 7 we show a particularly instructive observation, where a polished section was imaged twice at widely different frequencies, mid-infrared and far-infrared, in close sequence.

The mid-infrared and THz near-field images in Fig. 7 display both the two metal contact bars seen in topography, and the seven transistor structures in high scattering amplitude, and with high resolution of $40 \mathrm{~nm}$. In the Si substrate below the transistors no contrast is discernible in the mid infrared but surely in the $\mathrm{THz}$ image. This demonstrates and quantifies the doping gradient which was designed into the structure (according to the mobile-carrier density values indicated). This result very directly verifies the basic message given in Fig. 2, that infrared s-SNOM is well suited to characterize and even quantify Drude-like conduction, with particularly high sensitivity to low semiconductor doping when used in the $\mathrm{THz}$ region.

\section{Nanoscopy of non-Drude conduction phenomena}

In Fig. 4 we showd already that the non-Drude contribution to the optical conductivity of metals should lead to characteristic, strong s-SNOM signatures; these should allow an easy observation of metals because of the high scattering amplitude, and to a good discrimination between different metals because of the spectral richness. Similarly advantageous performance can be expected for many other classes of non-Drude conductors, for example, organic conductors, superconductors, or low-dimensional electron 


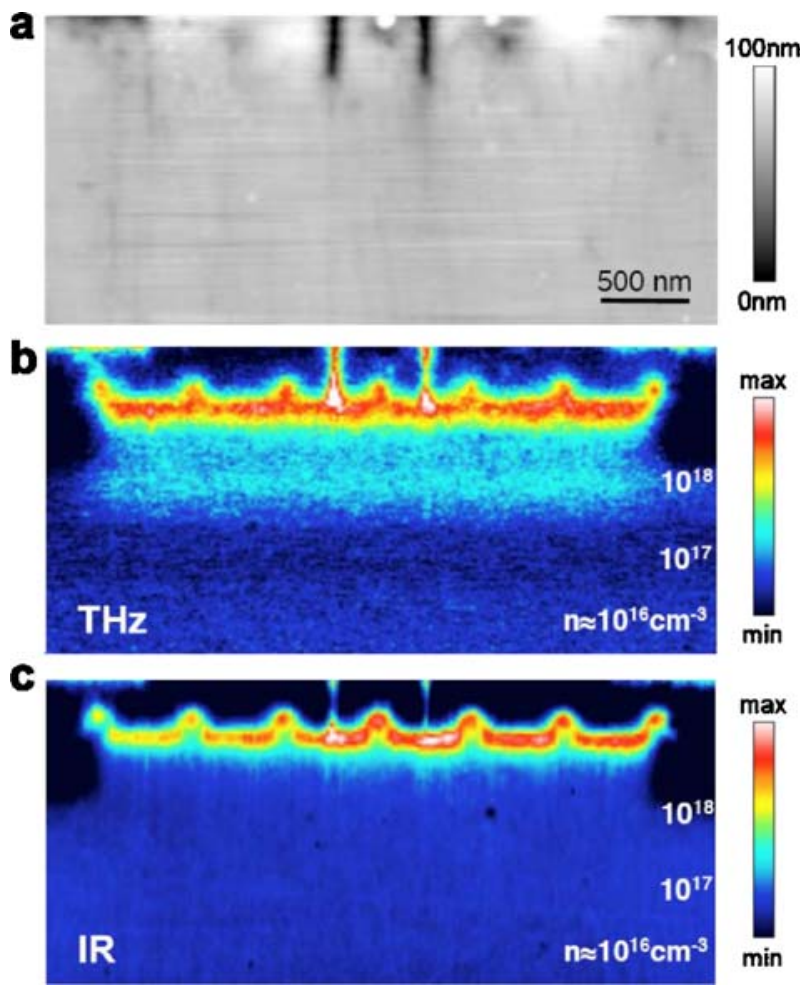

Fig. $71.7 \times 3.8 \mu \mathrm{m}^{2} \mathrm{~s}-\mathrm{SNOM}$ images of a polished cut through a multiple-transistor device structure of $65-\mathrm{nm}$ technology, adapted from [44]. (a) AFM topography and simultaneously acquired (b) THz and (c) mid-infrared near-field $s_{2}$ amplitude images, taken at $118 \mu \mathrm{m}$ and $11 \mu \mathrm{m}$ wavelength, respectively.

systems in specific frequency regions. Quite generally, the capability of s-SNOM to locally quantify the complex dielectric function enables to study such materials far beyond the level of a mere material recognition. Indeed the physics of the underlying conduction phenomenon can be explored.

As the first and recent example we present here a result taken with mid-infrared sSNOM of an interesting "correlated" metal which shows spontaneous nanoscale inhomogeneity (Fig. 8).

Crystalline $\mathrm{VO}_{2}$ is of high fundamental interest because it is an insulator at room temperature that turns metallic at the insulator-to-metal phase transition temperature near $343 \mathrm{~K}$. The nature of this phase transition has long been debated. Figure 8 shows a highly resolved near-field image of such a crystal at a temperature in the midst of the insulator-tometal phase transition. The surface has a slightly rough topography, indicating $\approx 100-\mathrm{nm}$ sized crystallites, but the infrared image shows larger, distinct conductive regions (red, strong scattering) embedded in insulating host material (blue, weak scattering). The conductive regions possess structural detail down to the resolution limit of about $20 \mathrm{~nm}$. They can be observed to grow with increasing temperature. Thus this material can be depicted as spontaneously nano-structured in its conductive property, and the character of its phase transition has been assigned to be of Mott-type [36, 45, 46].

Note that this result was obtained by imaging the sample at a single mid-infrared wavelength only. A detailed analysis yielded the further, highly interesting result that the 

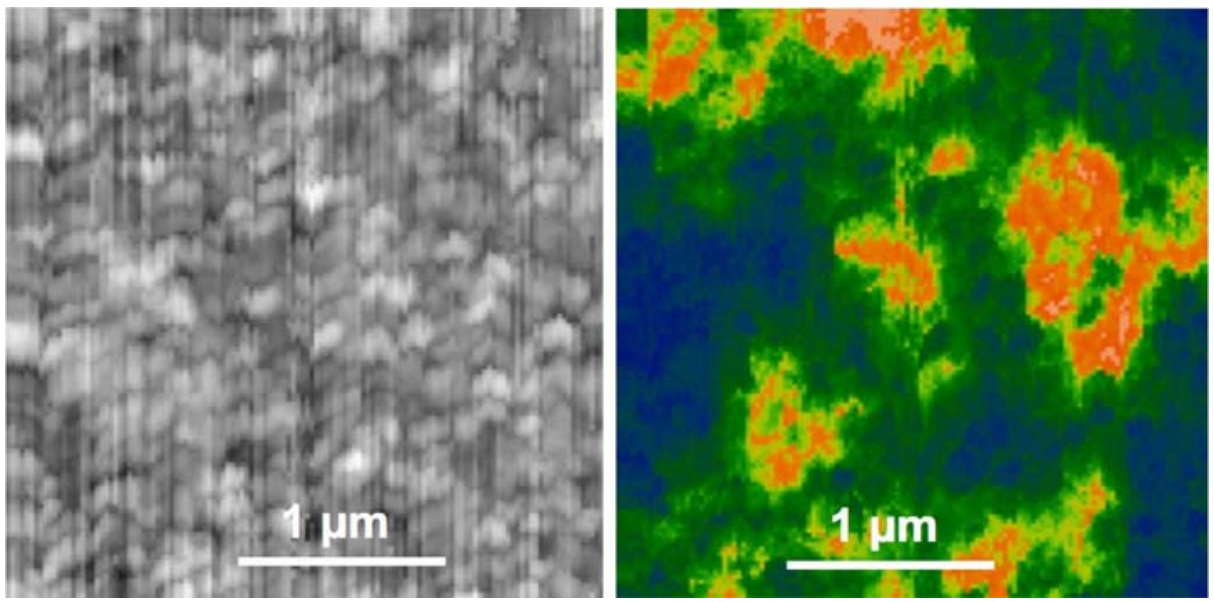

Fig. $83 \times 3 \mu \mathrm{m}^{2}$ mid-infrared s-SNOM $s_{2}$ amplitude image of a $\mathrm{VO}_{2}$ crystal at $343 \mathrm{~K}$, taken at $\lambda=10.6 \mu \mathrm{m}$, topography (left), infrared amplitude (right).

metal nanoscale grains contain quasiparticles with enhanced mass due to electron correlation. This analysis was made possible by the repeatability of s-SNOM imaging at different temperatures, and in addition, by the ellipsometric determination of the spatially averaged dielectric function. Future extension of the methodology would vary the wavelength in the range possible with the $\mathrm{CO}_{2}$ laser $(9-11 \mu \mathrm{m})$, and beyond.

\section{Outlook}

The prospect is bright for s-SNOM to serve as a basic characterization tool of nanostructured conducting materials. As this technique is noninvasive, does not require a special sample preparation, and tolerates modest surface topography, it should become a valuable tool in both basic and applied nanoscience areas dealing with metals and semiconductors. The demonstrated usability of all spectral ranges from microwaves to visible guarantees that most if not all conducting mechanisms in solid state physics can be explored. Especially, with the recent closing of the "THz gap" in s-SNOM operation this nanoscope is well capable of quantifying the conductivity of state-of-the-art nano-electronic devices, as well as for exploring exotic conduction mechanisms provided by heavy, correlated, Cooper-paired or spin-aligned electrons.

Apart from cases of highly resonant conductivity which may be found in ultrahighmobility low-dimensional electron systems, for example, the typically rather broad spectral features induced by conductivity would not require a continuous-broadband spectral coverage, rather, operating the s-SNOM at few frequencies of perhaps two per decade should quite suffice for many applications. Up to now the mid-infrared version of s-SNOM operated with the $\mathrm{CO}_{2}$ laser has the technical advantage of being easiest operatable.

Altogether, conductivity contrast is a valuable addition to the s-SNOM's already widely known application potential of chemical and material recognition in the nanosciences and in nanotechnology. 
Acknowledgements We acknowledge the fruitful cooperation with Bernhard Knoll, Thomas Taubner, Reinhard Guckenberger, Markus Brehm, Nenad Ocelic, Jesper Wittborn, Javier Aizpurua, Antonija Cvitcovic, Dimitri Basov, Hans-Georg von Ribbeck, Mumtaz Qazilbash, Thomas Ganz, Alexander Ziegler, and Thomas Koeck. We thank Frank Neubrech for supplying dielectric data. Supported by BMBF grant 3 N8705, and DFG Clusters of Excellence Nano-Initiative Munich and Munich-Centre for Advanced Photonics.

Open Access This article is distributed under the terms of the Creative Commons Attribution Noncommercial License which permits any noncommercial use, distribution, and reproduction in any medium, provided the original author(s) and source are credited.

\section{References}

1. L. Novotny, and B. Hecht, Principles of Nano-Optics (Cambridge, 2007).

2. J. Wessel, "Surface-enhanced optical microscopy," Journal of the Optical Society of America B 2, 15381540 (1985).

3. H. K. Wickramasinghe, and C. C. Williams, "Apertureless near field optical microscope," U.S. Patent, 4,947,034 (1990).

4. F. Keilmann and R. Hillenbrand, "Near-field nanoscopy by elastic light scattering from a tip," in NanoOptics and Near-Field Optical Microscopy, edited by A. Zayats and D. Richards (ISBN 978-1596932838 ArtechHouse, 2009), pp. 235-265.

5. M. Brehm, A. Schliesser, F. Cajko, I. Tsukerman, and F. Keilmann, "Antenna-mediated back-scattering efficiency in infrared near-field microscopy," Optics Express 16, 11203-11215 (2008).

6. N. Ocelic, A. Huber, and R. Hillenbrand, "Pseudo-heterodyne detection for background-free near-field spectroscopy," Applied Physics Letters 89, 101124-1-101124-3 (2006).

7. H. Kuzmany, Solid-State Spectroscopy (Springer, Berlin, 1998).

8. B. Knoll and F. Keilmann, "Near-field probing of vibrational absorption for chemical microscopy," Nature 399, 134-137 (1999).

9. R. Hillenbrand and F. Keilmann, "Complex optical constants on a subwavelength scale," Physical Review Letters 85, 3029-3032 (2000).

10. A. Cvitkovic, N. Ocelic, and R. Hillenbrand, "Analytical model for quantitative prediction of material contrasts in scattering-type near-field optical microscopy," Optics Express 15, 8550 (2007).

11. R. Hillenbrand, T. Taubner, and F. Keilmann, "Phonon-enhanced light-matter interaction at the nanometre scale," Nature 418, 159-162 (2002).

12. E. A. Ash and G. Nicholls, "Super-resolution aperture scanning microscope," Nature 237, 510-512 (1972).

13. F. Keilmann, D. W. van der Weide, T. Eickelkamp, R. Merz, and D. Stöckle, "Extreme sub-wavelength resolution with a scanning radio-frequency transmission microscope," Optics Communications 129, 1518 (1996).

14. B. Knoll, F. Keilmann, A. Kramer, and R. Guckenberger, "Contrast of microwave near-field microscopy," Applied Physics Letters 70, 2667-2669 (1997).

15. B. Rosner, J. Peck, and D. W. van der Weide, "Near-field antennas integrated with scanning probes for THz to visible microscopy: scale modelling and limitations on performance," IEEE Transactions on Antennas and Propagation 50, 670-675 (2002).

16. A. Imtiaz, S. M. Anlage, J. D. Barry, and J. Melngailis, "Nanometer-scale material contrast imaging with a near-field microwave microscope," Applied Physics Letters 90, 143106-1-143106-3 (2007).

17. K. Lai, H. Peng, W. Kundhikanjana, D. T. Schoen, C. Xie, S. Meister, Y. Cui, M. A. Kelly, and Z. X. Shen, "Nanoscale Electronic Inhomogeneity in In2Se3 Nanoribbons Revealed by Microwave Impedance Microscopy," Nanoletters. doi:10.1021/n19002

18. F. Zenhausern, M. P. O’Boyle, and H. K. Wickramasinghe, "Apertureless near-field optical microscope," Applied Physics Letters 65, 1623-1625 (1994).

19. M. B. Raschke and C. Lienau, "Apertureless near-field optical microscopy: tip-sample coupling in elastic light scattering," Applied Physics Letters 83, 5089-5091 (2003).

20. L. Stebounova, B. B. Akhremitchev, and G. C. Walker, "Enhancement of the weak scattered signal in apertureless near-field scanning infrared microscopy," Review of Scientific Instruments 74, 3670-3674 (2003). 
21. T. Taubner, F. Keilmann, and R. Hillenbrand, "Nanoscale-resolved subsurface imaging by scattering-type near-field optical microscopy," Optics Express 13, 8893-8899 (2005).

22. Z. H. Kim and S. R. Leone, "High-resolution apertureless near-field imaging using gold nanosphere probes," Journal of Physical Chemistry B 110, 19804 (2006).

23. R. Esteban, R. Vogelgesang, and K. Kern, "Tip-substrate interaction in optical near-field microscopy," Physical Review B 75, 195410-1-195410-8 (2007).

24. G. Wollny, E. Bründermann, Z. Arsov, L. Quaroni, and M. Havenith, "Nanoscale depth resolution in scanning near-field infrared microscopy," Optics Express 16, 7453-7459 (2008).

25. E. W. Palik, Handbook of Optical Constants of Solids (Academic Press, San Diego, 1985).

26. M. Brehm, A. Schliesser, and F. Keilmann, "Spectroscopic near-field microscopy using frequency combs in the mid-infrared," Optics Express 14, 11222-11233 (2006).

27. A. Lahrech, R. Bachelot, P. Gleyzes, and A. C. Boccara, "Infrared-reflection-mode near-field microscopy using an apertureless probe with a resolution of $\lambda / 600$," Optics Letters 21, 1315-1317 (1996).

28. B. Knoll and F. Keilmann, "Infrared conductivity mapping for nanoelectronics," Applied Physics Letters 77, 3980-3982 (2000).

29. R. Hillenbrand, B. Knoll, and F. Keilmann, "Pure optical contrast in scattering-type scanning near-field optical microscopy," Journal of Microscopy 202, 77-83 (2001).

30. R. Hillenbrand and F. Keilmann, "Material-specific mapping of metal/semiconductor/dielectric nanosystems at $10 \mathrm{~nm}$ resolution by back-scattering near-field optical microscopy," Applied Physics Letters 80, 25-27 (2002).

31. T. Taubner, R. Hillenbrand, and F. Keilmann, "Nanoscale polymer recognition by spectral signature in scattering infrared near-field microscopy," Applied Physics Letters 85, 5064-5066 (2004).

32. A. Cvitkovic, N. Ocelic, J. Aizpurua, R. Guckenberger, and R. Hillenbrand, "Infrared Imaging of Single Nanoparticles via Strong Field Enhancement in a Scanning Nanogap," Physical Review Letters 97, 60801-1-60801-4 (2006).

33. M. Brehm, T. Taubner, R. Hillenbrand, and F. Keilmann, "Infrared spectroscopic mapping of single nanoparticles and viruses at nanoscale resolution," Nano Letters 6, 1307-1310 (2006).

34. A. J. Huber, D. Kazantsev, F. Keilmann, J. Wittborn, and R. Hillenbrand, "Simultaneous infrared material recognition and conductivity mapping by nanoscale near-field microscopy," Advanced Materials 19, 2209-2213 (2007).

35. A. Cvitkovic, N. Ocelic, and R. Hillenbrand, "Material-specific infrared recognition of sub-10 nm particles by substrate-enhanced scattering-type near-field microscopy," Nano Letters 7, 3177-3181 (2007).

36. M. M. Qazilbash, M. Brehm, B. G. Chae, P. C. Ho, G. O. Andreev, B. J. Kim, S. J. Yun, A. V. Balatsky, M. B. Maple, F. Keilmann, H. T. Kim, and D. N. Basov, "Mott transition in VO2 revealed by infrared spectroscopy and nano-imaging," Science 318, 1750-1753 (2007).

37. K. Mueller, X. Yang, M. Paulite, Z. Fakhraai, N. Gunari, and G. C. Walker, "Chemical Imaging of the Surface of Self-Assembled Polystyrene-b-Poly(methyl methacrylate) Diblock Copolymer Films Using Apertureless Near-Field IR Microscopy,” Langmuir 24, 6946-6951 (2008).

38. M. B. Raschke, L. Molina, T. Elsaesser, D. H. Kim, W. Knoll, and K. Hinrichs, "Apertureless Near-Field Vibrational Imaging of Block-Copolymer Nanostructures with Ultrahigh Spatial Resolution," ChemPhysChem 6, 2197-2203 (2005).

39. G. Masetti, M. Severi, and S. Solmi, "Modeling of carrier mobility against carrier concentration in arsenic-, phosphorus-, and boron-doped silicon," IEEE Trans. Electron Devices, 764-769 (1983).

40. A. J. Huber, A. Ziegler, T. Köck, and R. Hillenbrand, "Infrared nanoscopy of strained semiconductors," Nature Nanotechnology 4, 153-157 (2009).

41. H. G. von Ribbeck, M. Brehm, D. W. van der Weide, S. Winnerl, O. Drachenko, M. Helm, and F. Keilmann, "Spectroscopic THz near-field microscope," Optics Express 16, 3430-3438 (2008).

42. H. T. Chen, R. Kersting, and G. C. Cho, "THz imaging with nanometer resolution,", Applied Physics Letters 83, 3009-3011 (2003).

43. F. F. Buersgens, H. T. Chen, and R. Kersting, "Terahertz microscopy of charge carriers in semiconductors," Applied Phyics Letters 88, 112115 (2006).

44. A. J. Huber, F. Keilmann, J. Wittborn, J. Aizpurua, and R. Hillenbrand, "Terahertz near-field nanoscopy of mobile carriers in single semiconductor nanodevices,", Nano Letters 8, 3766-3770 (2008).

45. T. Driscoll, S. Palit, M. M. Qazilbash, M. Brehm, F. Keilmann, B. G. Chae, H. T. Kim, S. Y. Cho, N. M. Jokerst, and D. N. Basov, "Dynamic tuning of an infrared hybrid-metamaterial resonance using VO2," Applied Physics Letters 93, 24101-1-24101-3 (2008).

46. M. M. Qazilbash, M. Brehm, G. O. Andreev, A. Frenzel, P. C. Ho, B. G. Chae, B. J. Kim, S. J. Yun, H. T. Kim, A. V. Balatsky, O. G. Shpyrko, M. B. Maple, F. Keilmann, and D. N. Basov, "Infrared spectroscopy and nano-imaging of the insulator-to-metal transition in vanadium dioxide," Physical Review B 79, 075107-1-075107-10 (2009). 